\title{
Very short-range wakefields in strongly tapered disk-loaded waveguide structures
}

\author{
A. Grudiev \\ CERN, CH-1211 Geneva-23, Switzerland \\ (Received 27 August 2012; published 4 December 2012)
}

\begin{abstract}
Very short-range wakefields in strongly tapered disk-loaded waveguide structures are investigated in the regime where the so-called "catch-up" distance is much larger than the length of the tapered structure. It is shown that in this case the very short-range wakefields are not being an average over all irises of the tapered structure; instead they are dominated by the irises with the smallest aperture radius. A new analytical model for short-range wakefield calculation in this regime is proposed. The results of the analytical model and numerical simulations are compared for the case of X-band accelerating structures.
\end{abstract}

DOI: 10.1103/PhysRevSTAB.15.121001

PACS numbers: 29.27.Bd

\section{INTRODUCTION}

Short-range wakefields are extremely important in the design of the linacs in general and linac based $x$-ray free electron lasers (FELs) and linear colliders in particular. Moreover, electron bunches are very short in linear colliders, and even shorter in linac based x-ray FELs. For example, in the SwissFEL project [1] the nominal rms bunch length is $\sigma_{z}=10 \mu \mathrm{m}$ and $2 \mu \mathrm{m}$ for the long and short pulse operation modes, respectively. In addition, typical disk-loaded waveguide structures used for charged particle acceleration are tapered. For example, in the Compact Linear Collider (CLIC) [2], in order to achieve highest accelerating gradient, the $\mathrm{X}$-band accelerating structure is chosen to have small aperture strongly tapered from $3.15 \mathrm{~mm}$ down to $2.35 \mathrm{~mm}$ over a distance of $\sim 230 \mathrm{~mm}$ only [3]. There is a great potential of using this type of high gradient accelerating structures for compact X-ray FELs. For example, in [4], it has been proposed to use X-band accelerating structures developed for the Next Linear Collider (NLC) project [5]. Furthermore, even more compact linacs can be designed based on the higher gradient accelerating structure developed for CLIC. On the other hand, in the design of the linacs in general and linac based x-ray FELs in particular, it is extremely important to calculate short-range wakefields accurately also in the case of strongly tapered accelerating structures where the effect of tapering may be significant.

A qualitative picture of the short-range longitudinal wake in an infinitely long periodic structure of disk-loaded waveguide is described in [6]. The wakefields form when perturbations of the "pancake"-like electromagnetic fields of an ultrarelativistic particle caused by an iris tip move towards the structure axis according to

Published by the American Physical Society under the terms of the Creative Commons Attribution 3.0 License. Further distribution of this work must maintain attribution to the author(s) and the published article's title, journal citation, and DOI.

$$
\delta \approx \sqrt{2 z s}
$$

where $\delta$ is the difference in the radial positions of the iris tip, which excites the wakefields, and the front of the wakefields, $z$ is the distance between the iris and the witness particle, and $s$ is the distance between the drive and witness particles. The distance covered by the witness particles from the moment when it passes the iris until the moment when the wakefields reach the witness particle is called "catch-up" (CU) distance [7]. It is expressed in terms of the number of cells to catch-up from Eq. (1) with $\delta=a$ :

$$
N_{\mathrm{CU}} \approx a^{2} / 2 L s,
$$

where $a$ and $L$ are the iris radius and the cell length, respectively.

The widely used Karl Bane's model (KB model) is proposed for calculation of the short-range longitudinal wake function for infinitely long periodic structures in [8]:

$$
W_{L}\left(s, s_{0}\right)=\frac{Z_{0} c}{\pi a^{2}} \exp \left(-\sqrt{s / s_{0}}\right) ; \quad \text { where } s_{0}=0.41 \frac{a^{1.8} g^{1.6}}{L^{2.4}},
$$

where $g$ is the gap between the irises and $Z_{0}$ and $c$ are the impedance and the speed of light in vacuum, respectively. In the same publication, the wakefield in a tapered NLC accelerating structure was calculated by averaging Eq. (3) over all the different 206 cells of the structure. This was justified since $N_{\mathrm{CU}}=14$ which is much less than the number of cells in the structure: $N_{c}=206$. The bunch length used for Eq. (2) is $s \approx \sigma_{z} \approx 120 \mu \mathrm{m}$. However, in CLIC main linac the situation is different. The aperture is smaller and the bunch is shorter, $\sigma_{z}=44 \mu \mathrm{m}$, resulting in the same $N_{\mathrm{CU}}=14$ which is comparable to a much shorter structure of $N_{c}=26$. This means the assumption that the wakefield "averages" inside the structure is not valid. Furthermore for X-ray FELs, the bunch length is even shorter, for example, $\sigma_{z}=10 \mu \mathrm{m}$ or even $\sigma_{z}=2 \mu \mathrm{m}$ in the case of SwissFEL [1]. Assuming such short bunches, $N_{\mathrm{CU}}$ is 60 and 300, respectively, which is (much) larger 
than the number of cells in a high gradient $\mathrm{X}$-band accelerating structure like the one for the CLIC main linac. In this regime, the wakefields from the irises with larger aperture are intercepted by the smaller irises which dominate the wakefields more and more as the bunch length approaches zero. Taking an extreme case of zero bunch length $(s \rightarrow 0)$, the irises with the smallest aperture $a_{\text {min }}$ completely suppress the wakefields from all the other irises so that for any tapered structure the averaging technique from [8] which results in the expression on the right-hand side of Eq. (4) always underestimates the wakefields compared to the correct result shown on the left-hand side of Eq. (4),

$$
W_{L}^{\mathrm{TS}}(0)=\frac{Z_{0} c}{\pi a_{\min }^{2}}>\left\langle W_{L}(0)\right\rangle=\frac{1}{N_{c}} \sum_{n=1}^{N_{c}} \frac{Z_{0} c}{\pi a_{n}^{2}},
$$

where $a_{n}$ are aperture radii of the irises. Moreover, for any tapered structure there is a threshold value for $s$ :

$$
s_{\mathrm{CU}} \approx a_{\mathrm{min}}^{2} / 2 L N_{c} \text {. }
$$

Below it for $s<s_{\mathrm{CU}}$ the very short-range wakefields cannot be obtained correctly by using the averaging technique from [8]. Instead more sophisticated models or simulations are required in order to accurately calculate very short-range wakefields in strongly tapered diskloaded waveguide structures.

Recently, a new expression for the wake function of the SLAC S-band constant gradient accelerating structures has been found based on the fitting of the wake potential of a very short bunch to simulation results [9]. It has been shown that the maximum value of the wake potential excited by a bunch of $\sigma_{z}=10 \mu \mathrm{m}$ in the SLAC S-band linac is $18 \%$ higher than the one calculated using averaging technique similar to [8]. This qualitatively agrees with our above considerations since in this case $N_{\mathrm{CU}}=240$ is larger than $N_{c}=86$. Since the new wake function expression found in [9] has two parameters which have been optimized to fit the simulation results for the SLAC S-band linac it cannot be used for the other geometries.

In this paper, a semianalytical model is proposed which gives lower and upper estimates of the wake function in the tapered structure as a function of its geometrical parameters.

\section{KB MODEL AND SIMULATIONS}

In Fig. 1, a sample structure geometry is shown with $L=10 \mathrm{~mm}, g=9 \mathrm{~mm}$, and an iris radius $a_{n}$ which is tapered from 4 to $2 \mathrm{~mm}$ over 26 cells. This structure has roughly the length of the CLIC main linac accelerating structure but stronger tapering in order to better demonstrate the effect of tapering. Nevertheless it is close enough to a practical structure like, for example, to one of the earlier X-band prototypes known as the T18 [10]. The short-range longitudinal wakefields have been calculated in two ways using the averaged $\mathrm{KB}$ model $(\langle\mathrm{KB}\rangle)$ and

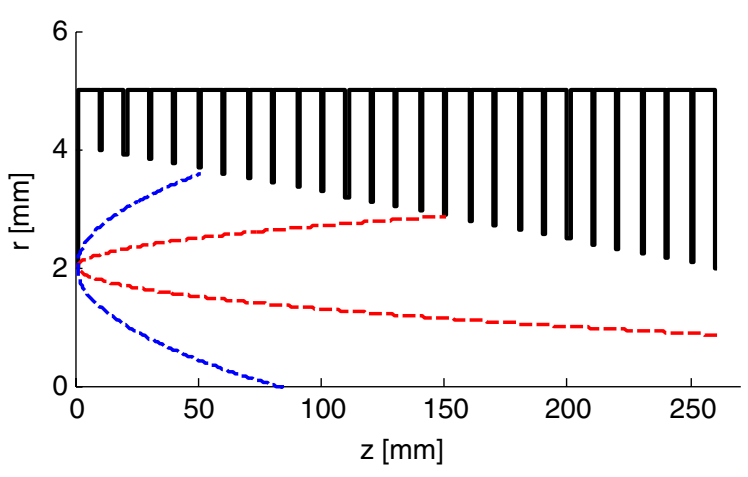

FIG. 1. Geometry of a sample structure is shown in the black solid line. Diffraction on the smallest iris is illustrated in red and blue dashed lines for different $s: 5$ and $50 \mu \mathrm{m}$, respectively.

numerically using the ECHO2D code [11]. For numerical simulations the tapered structure geometry presented in Fig. 1 has been repeated many times in order to arrive at the total length of the simulated structure much larger than the catch-up distance. This gives an asymptotic solution when the wakefields per meter length do not depend on the total length of the simulated structure. In our case, the criterion was that the change of the total loss factor per meter length is below 2 per mil when the total length is increased by factor 2 . Simulations have been performed for two different bunch lengths $\sigma_{z}: 10$ and $5 \mu \mathrm{m}$. The total numbers of structures were 32 and 200 , respectively. Simulations for shorter bunch require more computer resources due to both larger total length of the simulated structure and smaller mesh size. Numerical convergence has been checked as a function of mesh size and the following values were used: $d z=2 \mu \mathrm{m}$ for $\sigma_{z}=$ $10 \mu \mathrm{m}$ and $d z=1 \mu \mathrm{m}$ for $\sigma_{z}=5 \mu \mathrm{m}$. In both cases,

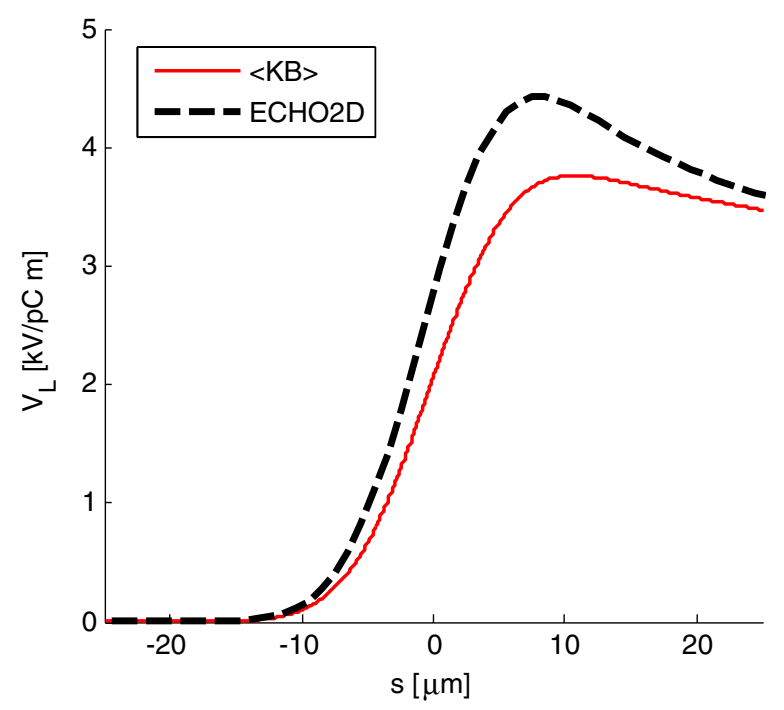

FIG. 2. Short-range longitudinal wake potential for a bunch of $\sigma_{z}=5 \mu \mathrm{m}$ in the sample structure (Fig. 1) were calculated using the $\langle\mathrm{KB}\rangle$ model (solid line) and the ECHO2D code (dashed line). 
$d r=5 d z$. It was not possible to simulate wakefields for $\sigma_{z}=2 \mu \mathrm{m}$ due to practical limitations of computer resources. The results for $\sigma_{z}=5 \mu \mathrm{m}$ are presented in Fig. 2 demonstrating a clear difference between the $\langle\mathrm{KB}\rangle$ model and the ECHO2D simulations. A difference of $30 \%$ in the total loss factor is observed.

Qualitatively, the effect can be explained as follows. As the witness particle comes closer and closer to the drive particle $(s \rightarrow 0)$, the catch-up distance increases and becomes larger than the tapered structure length. The divergence angle of the wakefields which can still catch-up with the witness particle becomes smaller because a larger part of the wakefield is intercepted by the next downstream iris with the smallest aperture. At some point all wakefields which would catch-up the witness particle in the case of no tapering are intercepted by the smallest iris except from the one which comes from the iris itself. According to Eq. (2), the larger the iris aperture radius the larger the catch-up distance. As it is illustrated in Fig. 1 the wakefield front moves away from the iris, not along a straight line like a monochromatic wave would do, but due to extremely broadband nature it follows a square root dependence on $z$ for a given $s$. That means that the wakefields from the largest iris will be suppressed first. Then, one by one depending on the iris radius, wakefields from all of the irises but the smallest one will be suppressed when approaching the extreme case of zero bunch length. In Fig. 1, the diffraction on the smallest iris is illustrated for different $s$ demonstrating that the smaller is $s$ the more irises remain in the shadow. Expressed in the terminology used in [12], the diffraction angle of the wakefields becomes smaller as their frequency gets higher at $s \rightarrow 0$ leading to the extreme case of diffraction only on the smallest irises spaced by the length of the tapered structure. All of the other irises remain in the "shadow" of the smallest irises. In fact, the situation becomes equivalent to periodic structure of longer cells with the following effective parameters: $a^{*}=$ $a_{\min } ; L^{*}=L N_{c} ; g^{*}=L\left(N_{c}-1\right)+g$. The short-range longitudinal wakefields in the periodic structure of long cells is calculated in the following section.

\section{CORRECTED KB MODEL FOR LONG CELL PERIODIC STRUCTURES}

The KB model is based on a semianalytical approach in which the model is fitted to a set of data obtained from numerical simulations [8]. It has its own applicability range: $0.34<a / L<0.69 ; 0.54<g / L<0.9$ which is related to the NLC structure design parameters. In the case of the very short-range wakefields in our sample structure shown in Fig. $1, a^{*} / L^{*}=0.008$ which is far outside the range of applicability of KB model. Dedicated simulations have been performed to study the short-range longitudinal wakefields in periodic structures of $a=2 \mathrm{~mm}$ and $L-g=1 \mathrm{~mm}$ with very small $a / L$. The results for $\sigma_{z}=5 \mu \mathrm{m}$ are presented in Fig. 3, clearly demonstrating

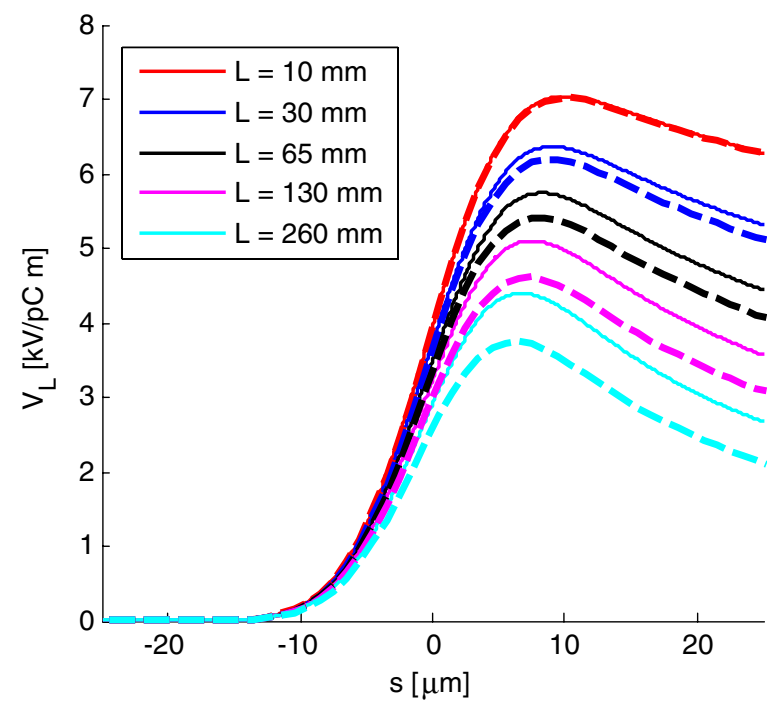

FIG. 3. Short-range longitudinal wake potentials for a bunch of $\sigma_{z}=5 \mu \mathrm{m}$ in a periodic structure of radius $a=2 \mathrm{~mm}$ were calculated using the $\mathrm{KB}$ model (solid lines) and the ECHO2D code (dashed lines) for different values of the cell length (see color code in the legend).

the difference between the $\mathrm{KB}$ model and the ECHO2D simulations for $a / L \ll 1$.

Corrections to the KB model have been found by fitting the ECHO2D simulation results at different iris radius $a$ of 1 and $2 \mathrm{~mm}$ for two different rms bunch lengths of 10 and $5 \mu \mathrm{m}$. A newly found expression similar to Eq. (3) with correction factors for $s_{0}$ follows:

$$
W_{L}^{*}=W_{L}\left(s, s_{0}^{*}\right) ; \quad \text { where } s_{0}^{*}=s_{0} \cdot 1.3\left(\frac{a}{L}\right)^{0.14} .
$$

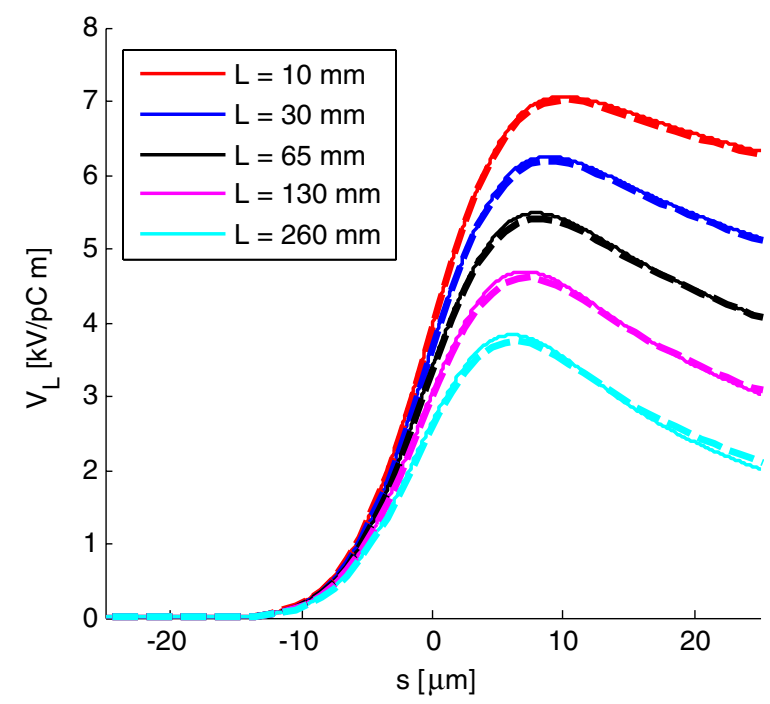

FIG. 4. Short-range longitudinal wake potentials for a bunch of $\sigma_{z}=5 \mu \mathrm{m}$ were calculated using the $\mathrm{KB}^{*}$ model (solid lines) and the ECHO2D code (dashed lines) in the periodic structure of radius $a=2 \mathrm{~mm}$ for different values of $L$ (see color code in the legend). 


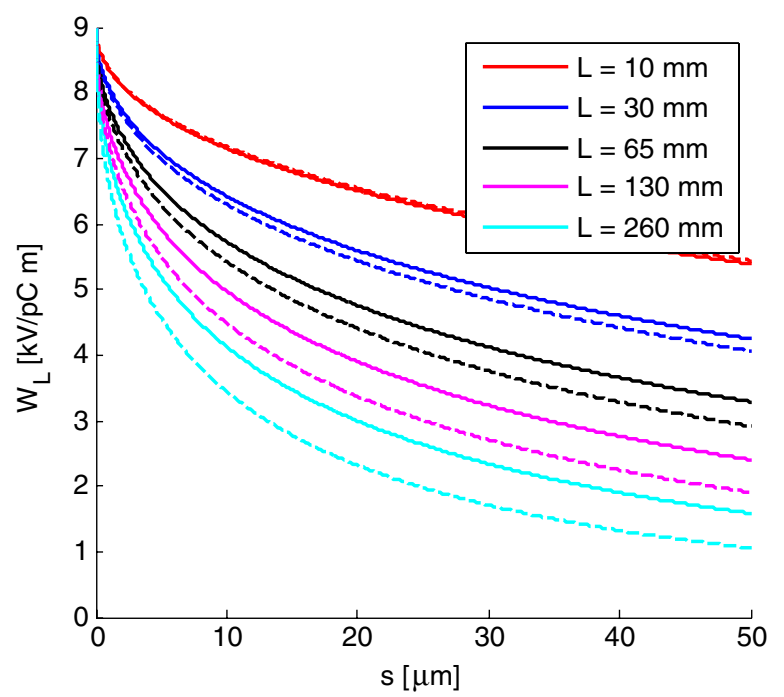

FIG. 5. Short-range longitudinal wake functions were calculated using $\mathrm{KB}$ (solid lines) and $\mathrm{KB}^{*}$ (dashed lines) models in the periodic structure of radius $a=2 \mathrm{~mm}$ for different values of $L$ (see color code in the legend).

Correction factors become significant only well outside of the original applicability range of the $\mathrm{KB}$ model. Inside of the applicability range of the KB model the difference between Eq. (3)and Eq. (6) is negligible. Comparison of the corrected $\mathrm{KB}$ model $\left(\mathrm{KB}^{*}\right)$ with the ECHO2D simulations is presented in Fig. 4.

In addition, in Fig. 5, wake functions calculated using $\mathrm{KB}$ and $\mathrm{KB}^{*}$ models are shown for comparison.

\section{NEW MODEL FOR THE VERY SHORT-RANGE WAKEFIELDS IN TAPERED STRUCTURES}

Finally, a new semianalytical model for the very shortrange wakefields in strongly tapered structures is presented in this section. The idea is based on the qualitative picture elaborated at the end of Sec. II. Assume that for a given $s$ there is an iris number $m$, which divides a tapered structure into two parts. The first part, with larger irises, from 1 to $m-1$ remains in the shadow of the smaller irises, which constitute the second part of the structure from $m$ to $N_{c}$. For example, as illustrated in Fig. 1, for $s=5$ and $50 \mu \mathrm{m}, m$ is equal to 15 and 5, respectively. Following this, the wake function expression is constructed from two parts corresponding to the wakes from the two parts of the tapered structure just described:

$$
\begin{aligned}
W_{L}^{(m)}(s)= & \frac{1}{N_{c}}\left[m \cdot W_{L}^{*}\left[a_{\min }, g+L(m-1), m L, s\right]\right. \\
& \left.+\sum_{n=m}^{N_{c}-1} W_{L}\left(a_{n}, g, L, s\right)\right],
\end{aligned}
$$

where the first term corresponds to the wake from the first part of the structure calculated using Eq. (6) with an effective length of the cells equal to the total length of the first part: $m L$; the second term is the wake from the second part calculated using standard averaging technique from [8]. There is a weighting factor $m$ for the first term to take into account the different effective cell length in the first and the second terms. Furthermore, $a_{\min }$ is used in the first part for the wake function calculation using Eq. (6). This is not quite correct since it is true only on one side of the first part and on the other side the iris radius changes depending on $m$. Nevertheless, the shadowing effect is dominated by the smallest irises of radius $a_{\min }$ and Eq. (7) gives a good upper estimate. Finally, two models for the short-range wake calculation in tapered structures are introduced:

Model $0(\mathrm{M} 0): W_{L}^{M 0}(s)$

$$
=\max \left\{W_{L}^{*}\left(a_{\min }, g^{*}, L^{*}, s\right),\left\langle W_{L}\left(a_{n}, g, L, s\right)\right\rangle\right\}
$$

and

Model $1(\mathrm{M} 1): W_{L}^{M 1}(s)=\underbrace{\max }_{m=1 \ldots N_{c}}\left\{W_{L}^{(m)}(s)\right\}$.

In Fig. 6, different short-range longitudinal wake functions are presented for comparison and discussion. First, wake functions for the largest and smallest irises spaced by $L$ are plotted to indicate the range of the wakes. Second, the wake function of the tapered structure calculated using the $\langle\mathrm{KB}\rangle$ model (red solid line) is compared to the wake function of the smallest irises spaced by the structure length $L N_{c}$ (red dashed line). These two curves intersect at $s=s_{\mathrm{CU}}=8 \mu \mathrm{m}$, estimated using Eq. (5) for the parameters under study. For $s>8 \mu \mathrm{m}$ the $\langle\mathrm{KB}\rangle$ model wake function is larger and dominates the wake, but for $s<8 \mu \mathrm{m}$ it underestimates the wake which is dominated

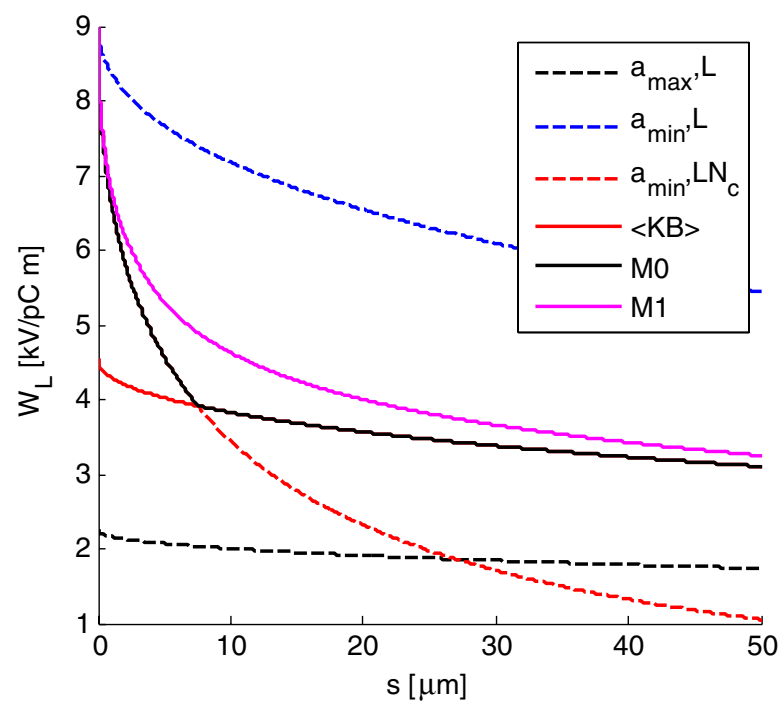

FIG. 6. Short-range longitudinal wake functions were calculated using the $\mathrm{KB}^{*}$ model in the periodic structures (dashed lines): first cell (black), last cell (blue), and last iris spaced by $L N_{c}$ (red); and using different models for the tapered structure (solid lines): $\langle\mathrm{KB}\rangle$ (red), M0 (black), M1 (magenta). 


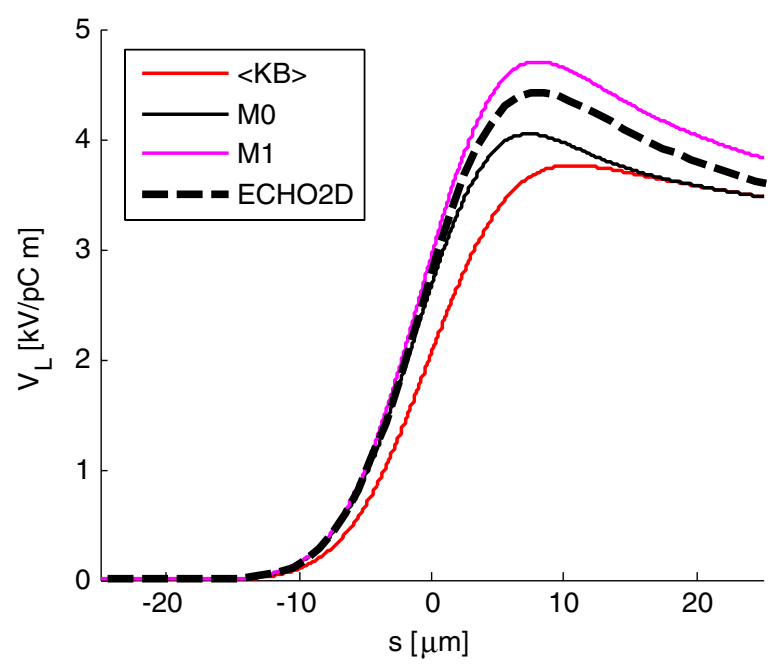

FIG. 7. Short-range longitudinal wake potentials were calculated for a bunch of $\sigma_{z}=5 \mu \mathrm{m}$ using different models (solid lines): $\langle\mathrm{KB}\rangle$ (red), M0 (black), M1 (magenta), and ECHO2D code (dashed line).

by the smallest irises as discussed above. Model 0 (M0) is the simplest model which just takes the maximum of these two curves in order to satisfy the known asymptotic solutions: $W_{L}^{(m=1)}$ for $s \ll s_{\mathrm{CU}}$ and $W_{L}^{(m=N c)}$ for $s \gg s_{\mathrm{CU}}$. It is likely too simplistic to adequately describe the wake function near the transition region: $s \sim s_{\mathrm{CU}}$. Nevertheless, it gives a lower estimate of the effect which is anyway larger than the $\langle\mathrm{KB}\rangle$ model for $s<s_{\mathrm{CU}}$. Model 1 (M1) is more elaborate. It is a maximum of Eq. (7) with $m$ running over all cells from 1 to $N_{c}$. It basically means that there are $N_{c}$ configurations each defining a range of $s$ for which the witness particle "sees" the corresponding iris range only. For each of the range of $s$ the iris configuration giving the maximum wake is taken. Both M0 and M1 have been investigated and compared to the ECHO2D simulation results. M1 provides a smoother transition at $s \sim s_{\mathrm{CU}}$ and indeed better agreement with the ECHO2D simulations results than M0, as shown in Fig. 7. It is worth mentioning that M1 gives an upper estimate for two reasons. First, as has already been mentioned above, Eq. (7) is an upper estimate by itself and second, at each $s$ maximum wakefield is taken in Eq. (9). Thus, M0 and M1 give lower and upper estimates of the very short-range wake function.

In order to illustrate the differences between the models quantitatively, Table I summarizes the total loss factor

TABLE I. Total loss factor per meter of length calculated using different models and ECHO2D in the sample structure for different bunch length is presented in $[\mathrm{kV} / \mathrm{pC} \mathrm{m}]$.

\begin{tabular}{lcccc}
\hline \hline$\sigma_{z}[\mu \mathrm{m}]$ & 2 & 5 & 10 & 44 \\
\hline ECHO2D & $\ldots$ & 2.63 & 2.32 & $\ldots$ \\
$\langle\mathrm{KB}\rangle$ & 2.11 & 2.02 & 1.93 & 1.62 \\
$\mathrm{M} 1$ & 3.19 & 2.78 & 2.46 & 1.81 \\
\hline \hline
\end{tabular}

calculated using different models and compares them to ECHO2D results when they can be simulated. There is clearly a significant difference between the $\langle\mathrm{KB}\rangle$ model and the ECHO2D simulation results. In addition, the table shows good agreement between $\mathrm{M} 1$ and ECHO2D results.

\section{VERY SHORT-RANGE TRANSVERSE WAKEFIELDS}

In [13], an expression for the short-range transverse (dipole) wake function for the periodic structure of cells with parameters defined as for Eq. (3) has been found for the same applicability range:

$W_{x}\left(s, s_{1}\right)=\frac{4 Z_{0} c}{\pi a^{4}} s_{1}\left[1-\left(1+\sqrt{s / s_{1}}\right) \exp \left(-\sqrt{s / s_{1}}\right)\right] ;$

where $s_{1}=0.169 \frac{a^{1.79} g^{0.38}}{L^{1.17}}$.

In order to obtain the transverse wake function for the tapered structure, the same averaging techniques as for the longitudinal wake function used in [8] have been applied to the case of NLC accelerating structures. All the arguments discussed above for the case of the very short-range longitudinal wakefields in the tapered structure apply to the case of the transverse wakefields. Thus, corrections to Eq. (10) have been found in the same way as for Eq. (3). A newly found expression with correction factors follows:

$$
W_{x}^{*}=W_{x}\left(s, s_{1}^{*}\right) ; \quad \text { where } s_{1}^{*}=s_{1} \cdot 1.3\left(\frac{a}{L}\right)^{0.28} .
$$

It is interesting to note that it has the same factor 1.3 as in Eq. (6) and the power factor 0.28 is 2 times larger than in Eq. (6). Following this, the transverse wake function expression is constructed in the same way as Eq. (7):

$$
\begin{aligned}
W_{x}^{(m)}(s)= & \frac{1}{N_{c}}\left[m \cdot W_{x}^{*}\left[a_{\min }, g+L(m-1), m L, s\right]\right. \\
& \left.+\sum_{n=m}^{N_{c}-1} W_{x}\left(a_{n}, g, L, s\right)\right] .
\end{aligned}
$$

Finally, Eqs. (10)-(12) are used in the models: M0 and M1 for the short-range transverse wake calculation in tapered structures:

Model 0 (M0): $W_{x}^{M 0}(s)$

$$
=\max \left\{W_{x}^{*}\left(a_{\min }, g^{*}, L^{*}, s\right),\left\langle W_{x}\left(a_{n}, g, L, s\right)\right\rangle\right\}
$$

Model $1(\mathrm{M} 1): W_{x}^{M 1}(s)=\underbrace{\max }_{m=1 \ldots N_{c}}\left\{W_{x}^{(m)}(s)\right\}$.

In Fig. 8, different short-range transverse wake functions are presented for comparison. In Fig. 9, results of $\langle\mathrm{KB}\rangle, \mathrm{M} 0$ and M1 models are compared to the ECHO2D simulations results indeed indicating that M1 give an upper estimate of 


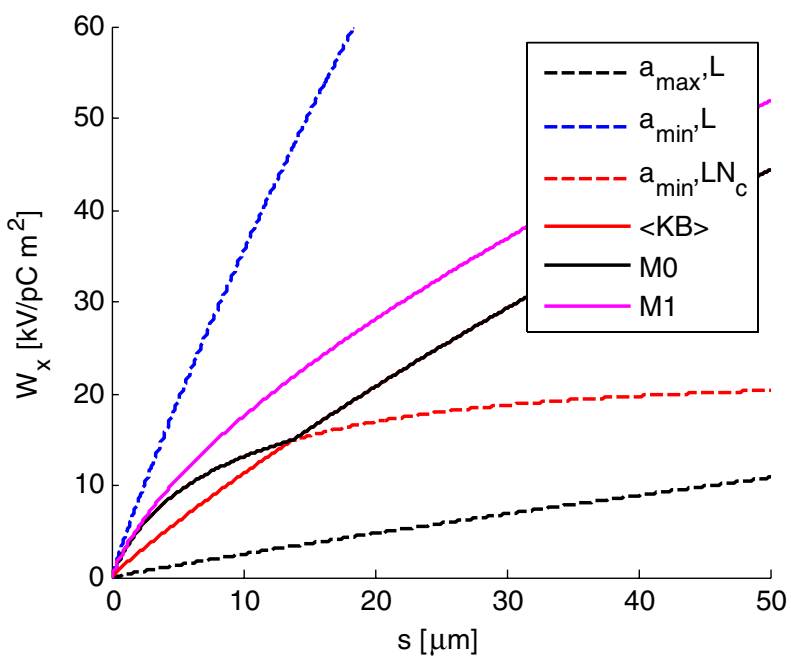

FIG. 8. Short-range transverse wake functions were calculated using the $\mathrm{KB}^{*}$ model in the periodic structures (dashed lines): first cell (black), last cell (blue), and last iris spaced by $L N_{c}$ (red); and using different models for the tapered structure (solid lines): $\langle\mathrm{KB}\rangle$ (red), M0 (black), M1 (magenta).

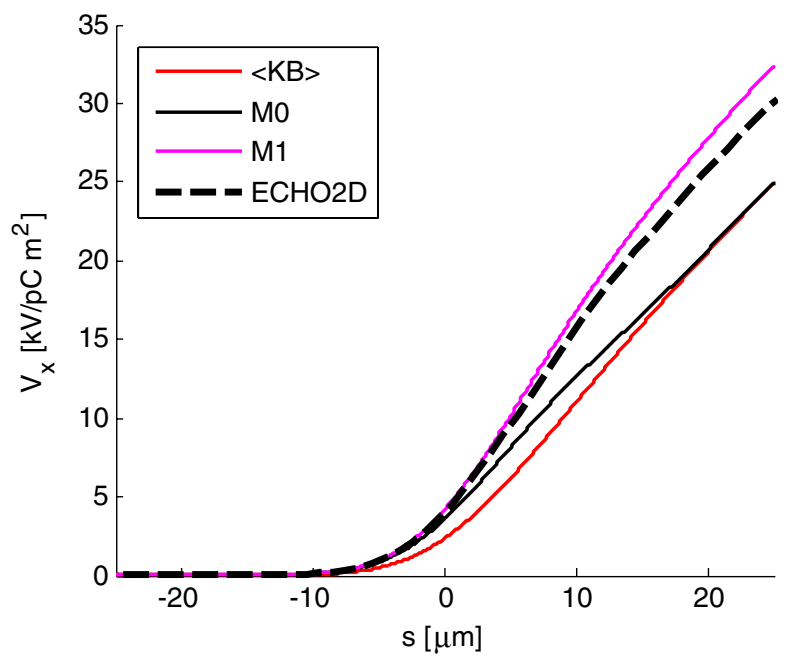

FIG. 9. Short-range longitudinal wake potentials were calculated for a bunch of $\sigma_{z}=5 \mu \mathrm{m}$ using different models (solid lines): $\langle\mathrm{KB}\rangle$ (red), M0 (black), M1 (magenta), and ECHO2D code (dashed line).

the very short-range transverse wake and is in a good agreement with the ECHO2D simulation results.

Table II summarizes the total kick factor calculated using different models and compares them to ECHO2D results if available. Again, there is a significant difference between the $\langle\mathrm{KB}\rangle$ and the ECHO2D simulation results as well as a good agreement between $\mathrm{M} 1$ and ECHO2D results.

\section{SUMMARY}

Effect of tapering in the disk-loaded waveguide structure on the very short-range longitudinal and transverse wake-
TABLE II. Total kick factor per meter of length calculated using different models and ECHO2D in the sample structure for different bunch length is presented in $\left[\mathrm{kV} / \mathrm{pC} \mathrm{m}^{2}\right]$.

\begin{tabular}{lcccc}
\hline \hline$\sigma_{z}[\mu \mathrm{m}]$ & 2 & 5 & 10 & 44 \\
\hline $\mathrm{ECHO} 2 \mathrm{D}$ & $\cdots$ & 5.12 & 8.25 & $\ldots$ \\
$\langle\mathrm{KB}\rangle$ & 1.37 & 3.21 & 5.99 & 20.5 \\
$\mathrm{M} 1$ & 2.73 & 5.38 & 8.77 & 23.8 \\
\hline \hline
\end{tabular}

fields is investigated. Depending on the bunch length and the structure parameters, significant discrepancy has been found between the widely used Karl Bane's model $[8,13]$ and numerical simulations using ECHO2D code [11] in the regime of the very short-range wakefields when $s<s_{\mathrm{CU}}$. A correction to Bane's model for periodic structures with small $a / L$ has been found and a new model for the lower and upper estimates of the very short-range wakefields in a tapered structure has been developed. Both the correction and the new model show good agreement with simulation results.

\section{ACKNOWLEDGMENTS}

The author is grateful to I. A. Zagorodnov for providing ECHO2D code and to $\mathrm{W}$. Wuensch for his careful reading the manuscript and useful comments.

[1] PSI Bericht Report No. 10-04, edited by R. Ganter, 2012.

[2] CLIC-Note 764, edited by F. Tecker, 2008.

[3] A. Grudiev and W. Wuensch, in Proceedings of the 25th Linear Accelerator Conference, Tsukuba, Japan, 2010, pp. 211-213.

[4] C. Adolphsen et al., in Proceedings of the 25th Linear Accelerator Conference, Tsukuba, Japan, 2010, pp. $428-430$.

[5] NLC ZDR Design Group, Technical report, SLAC Report No. 474, 1996.

[6] R. B. Palmer, Part. Accel. 25, 97 (1990).

[7] P. B. Wilson, Report No. SLAC-PUB-4547, 1989.

[8] K. Bane et al., in Proceedings of the 6th European Particle Accelerator Conference, Stockholm, 1998 (IOP, London, 1998).

[9] A. Novokhatski, Nucl. Instrum. Methods Phys. Res., Sect. A 684, 46 (2012).

[10] R. Zennaro et al., in Proceedings of 24th Linear Accelerator Conference, Victoria, BC, Canada, 2008, pp. 533-535.

[11] I. A. Zagorodnov and T. Weiland, Phys. Rev. ST Accel. Beams 8, 042001 (2005).

[12] G. V. Stupakov, in Proceedings of the Particle Accelerator Conference, Dallas, TX, 1995 (IEEE, New York, 1995), p. 3303.

[13] K. Bane, Report No. SLAC-PUB-9663, 2003. 